\title{
Long-term versus short-term consistency in the grapheme-colour synaesthesia: Grapheme-colour pairings can change in adulthood
}

\author{
Jan Chromý ${ }^{1}$ (1) Marianna Borůvková ${ }^{1} \cdot$ Lucie Malá $^{1} \cdot$ Tereza Sudzinová $^{1}$
}

Published online: 20 March 2019

(C) The Psychonomic Society, Inc. 2019

\begin{abstract}
The aim of the article is to examine the stability of grapheme-colour matchings in adulthood. We carried out a panel study using computerized tests to measure short-term and long-term consistency. We conducted three testing rounds during 1 year and a half with a resulting sample of 26 synaesthetes. Our results suggest that the colours associated with individual graphemes can change substantially (e.g. from "salmon" to "aquamarine") even during this relatively short time period. Based on a linear mixed model, we further argue that short-term and long-term consistency should be distinguished because these scores tend to differ. These results thus complement previous findings on the issue of grapheme-colour consistency and have important consequences for the study of synaesthesia in the future.
\end{abstract}

Keywords Synaesthesia $\cdot$ Colour $\cdot$ Grapheme-colour consistency $\cdot$ Crossmodality

People with grapheme-colour synaesthesia associate letters and/ or digits with colours. For example, for a grapheme-colour synaesthete, $M$ may be blue and 7 may be purple. A grapheme-colour synaesthete may associate a colour with each grapheme or just with some of them (Simner, 2007), and the colour may be either experienced "in the mind's eye" (socalled associators) or "seen" in external space or superimposed on the grapheme (so-called projectors; cf. Dixon, Smilek, \& Merikle, 2004; Ward, Li, Salih, \& Sagiv, 2007). Graphemecolour synaesthesia is a rare condition - the estimates are typically between $1 \%$ and $2 \%$ of the general population (cf. Carmichael, Down, Shillcock, Eagleman, \& Simner, 2015; Simner et al., 2006).

Grapheme-colour synaesthetes are typically identified based on the consistency of their grapheme-colour matchings. Consistency has been a central concept in synaesthesia research since at least the 1990s (cf. Baron-Cohen, 1996;

Electronic supplementary material The online version of this article (https://doi.org/10.3758/s13414-019-01707-6) contains supplementary material, which is available to authorized users.

Jan Chromý

jan.chromy@ff.cuni.cz

1 Institute of the Czech Language and Theory of Communication, Faculty of Arts, Charles University, Prague, Czech Republic
Baron-Cohen, Harrison, Goldstein, \& Wyke, 1993), and it has become one of the most widely used criteria for diagnosing grapheme-colour synaesthesia. It is generally accepted that grapheme-colour synaesthetes associate colours with graphemes consistently, whereas the consistency of grapheme-colour matchings for the rest of the population is lower (e.g. Simner et al., 2005; Ward \& Simner, 2005).

Grapheme-colour consistency has been measured using verbal responses (e.g. Baron-Cohen et al., 1993; Simner et al., 2005), printed colour swatches (e.g. Asher, Aitken, Farooqi, Kurmani, \& Baron-Cohen, 2006), and computerized tests (e.g. Simner, Harrold, Creed, Monro, \& Foulkes, 2009; Watson et al., 2017). Each of these ways of testing consistency has its own merits and limitations. It is complicated to assess consistency based on verbal responses (should "salmon" and "orange" be counted as a consistent response?), and the depth of analysis is limited because verbal responses do not offer precise information on the colour (e.g. its RGB values). Printed swatches may present a better method, but they are hardly ever used. Computerized tests have been a common way of testing consistency over the past 10 years, most likely because synaesthetes may be tested online without visiting the lab, tests are easily done, and, among other things, they offer precise information on the colour. The most widely used test for grapheme-colour synaesthesia is included in The Synesthesia Battery (http://synesthete.org/; Eagleman, Kagan, Nelson, Sagaram, \& Sarma, 2007). 
Consistency tests can be further classified based on the number of testing sessions. One possibility is to have two sessions. Participants are then instructed to match the list of graphemes to colours, whereupon there is a time gap (typically several months; cf. Rich, Bradshaw, \& Mattingley, 2005; Simner et al., 2005), and then they do the same grapheme-colour matching again. Based on the similarity of responses in the first and the second trial, the "long-term" consistency is calculated (a percentage of consistent matchings). Long-term consistency tests typically use verbal responses (i.e. verbal descriptions of perceived colours). The second possibility is to have only one session. Graphemes are presented in a randomized order, each grapheme is presented three times, and participants' task is to assign a colour to each grapheme on a colour palette. Differences between the colours for individual graphemes are calculated, and each participant's overall "short-term" consistency score is counted (see Rothen, Seth, Witzel, \& Ward, 2013, for various measures). These one-session measures typically use computerized testing. Thus, "short-term" consistency is measured within one testing session, whereas "long-term" consistency is measured within (at least) two temporally distant sessions.

It is widely accepted that the grapheme-colour matchings in adulthood are stable and long-lasting (cf. Carmichael et al., 2015). The idea of stability comes from studies that tested long-term consistency. For example, Simner (2012a) claims that "the typical synaesthete is around $80-100 \%$ consistent over time and controls are around $20 \%$ consistent" (p. 7). Importantly, the concept of consistency and its position in the research on synaesthesia has been disputed lately. The discussion has been primarily focused on the fact that there may be people who experience synaesthetic associations without being highly consistent in the tests (cf. Eagleman, 2012; Simner, 2012a, 2012b). The tentative conclusion is that people who are highly consistent in their associations present only a subset of the overall synaesthetic population.

Nevertheless, even if we consider only this subset as has been typically done, there are certain issues we should deal with. Indeed, long-term consistency of, say, $90 \%$ seems to be high (compared with typical non-synaesthetic scores), but this still means that 1 grapheme out of 10 is not associated with a colour consistently. This may be due to a measurement error (such as mouse-click mistakes in the online battery, low vividness of the colour for the particular synaesthete), but it is also possible that the grapheme-colour pairings may change during a lifetime. Indeed, this possibility is supported from other sources, such as self-reports. For example, Rich et al. (2005) claim that " $6 \%$ of respondents believed that their synaesthetic experiences were not consistent over time" (p. 67). And the present study was originally inspired by the synaesthete M. H., who claimed that her letter $T$ had suddenly turned black and acquired a velvety texture and that her letter $J$ had changed from yellow to orange.
The idea that the grapheme-colour matchings may not be stable during the lifetime is somehow supported by several recent studies. Kay, Carmichael, Ruffell, and Simner (2015) showed that the luminance of colours associated with graphemes decreases if the synaesthetes are in a negative mood. Meier, Rothen, and Walter (2014) measured graphemecolour consistency of 439 synaesthetes (divided into three age groups: younger age 18-28 years, middle age 29-42 years, and older age 43-91 years) and found that there is an age-related small but consistent decline in the number of consistent grapheme-colour associations. And recently, Simner, Ipser, Smees, and Alvarez (2017) compared synaesthetes from two age groups (below and above 35 years of age) and showed that synaesthetic colours become less saturated in aging, which causes lower synaesthetic consistency levels in older people. The latter two studies thus suggest that the graphemecolour associations change during a lifetime. However, the results are based on a comparison between age groups, and there is no direct evidence of an actual change in the grapheme-colour associations in individual synaesthetes.

The aim of the present study is to examine the stability of grapheme-colour matchings in adults systematically. We conducted a longitudinal panel study using computerized tests to measure and compare short-term and long-term consistency. To our knowledge, no study of this type has been carried out so far.

Our results show that the colours associated with graphemes in grapheme-colour synaesthesia can change substantially even during a relatively short time period. These results thus complement previous findings on the issue of graphemecolour consistency and have important consequences for the study of synaesthesia in the future.

\section{Material and method}

\section{Participants}

Synaesthetes were invited to participate in the research by means of posters distributed at the Charles University and various other places (libraries, cafeterias). Also, participants from former studies were invited to take part in this research.

Altogether, 42 self-declared adult synaesthetes expressed their willingness to participate in the study. In the first round, we tested all of them. Twenty-eight participants from the initial group were tested again in the second round. The rest of the participants did not continue in the second round although we contacted them via an email several times. In the third round, all the participants of the second round were tested again, except for one who did not respond at all. Altogether, 27 participants ( 25 women, two men; 23 Czech, two Slovak, one English, one Russian; median age during the first round = 25 years, first quartile $=23$ years, third quartile $=32$ years, 
min. 20, max. 48 years) were tested in all three rounds. One of them claimed to have only letter-colour synaesthesia and was not tested for digit-colour synaesthesia. All the other participants were tested for both letter-colour and digit-colour synaesthesia.

The participants received a reward of 300 CZK each (about 14 USD) for their participation in each round. For data anonymization, each participant was assigned a unique pseudonym.

\section{Materials and procedure}

The study consisted of three testing rounds which were conducted during a period of 16 months: The first round was conducted from the end of September till the first half of November 2015, the second round from the end of January 2016 till the beginning of April 2016, and the third round from the end of November 2016 till the first half of January 2017.

The participants were tested online using their own computers. Each round consisted of two tasks. The first task was to answer several questions. The main goal was to establish what computer the participants were using during the testing because we wanted to find out whether they were using the same or a different computer in all the rounds. As various screens display colours differently, the participants were strongly encouraged to use the same computer for all the rounds before the study started, and they were always reminded about that before each round. The questions in the first round also asked about some general characteristics of the participants, such as their native language and gender.

The second task consisted of the standardized digit-colour and Czech letter-colour consistency tests (www.synesthete. org; Eagleman et al., 2007). The digit-colour consistency test shows digits $0-9$ three times in a randomized order, and participants' task is to choose a colour from a palette which is the closest to the colour they associate with that digit. If they do not associate a colour with the digit, they may choose "no colour" response. The same procedure was adopted in the Czech letter-colour consistency test. The only difference was that instead of digits, the letters of the Czech alphabet were used. In the standardized battery, there are 38 letters of the Czech alphabet (A, Á, B, C, Č, D, Ď, E, É, Ě, F, G, H, I, Í, J, K, L, M, N, Ň, O, Ó, P, R, ̌̌, S, Š, T, ̌̌, U, Ú, Ů, V, Y, Ý, Z, and $\mathrm{Z}$ ). Unfortunately, the $\mathrm{Czech}$ digraph $\mathrm{CH}$ is not included in the Czech alphabet colour consistency test, and neither are the low-frequent letters Q, W, and X which occur in Czech loan words.

To undergo the tests in the battery, participants received a new login and password for each round. They could not access their results from the previous round, because passwords were changed after the completion of each round. This procedure was adopted to avoid problems with interconnecting the results of individual participants in the three rounds.

\section{Analysis}

The output of the consistency tests in the battery were RGB values for each grapheme-colour pairing. Because it has been shown that RGB distances are not ideal for measuring consistency (Rothen et al., 2013), we used CIELAB scores. The RGB values were converted to CIELAB values using the procedure specified in Rothen et al. (2013, p. 158). Thus, we linearized RGB values by applying standard gamma function on the RGB values using Formula 1:

$$
\begin{aligned}
\text { (i) } R G B l= & \frac{1}{1+0.055} \times(R B G g+0.055)^{2.4} \text { for discrete } R G B g(1) \\
& >0 \text { and (ii) } R G B l=0 \text { for discrete } R G B g=0,
\end{aligned}
$$

where $\mathrm{RGBl}=$ linear $\mathrm{RGB}, \mathrm{RGBg}=$ gamma-distributed $\mathrm{RGB}$, and RGB corresponds to R, G, B values.

Resulting values were then converted into $\mathrm{XYZ}$ values using the "RGB2XYZ" function in the "colorscience" package in R (Gama \& Davis, 2018), and from these values, CIELAB values were calculated using the "convertColor" function in the same package.

The distance between two colours of a grapheme was measured using the standard Euclidean metric: for two CIELAB colours $\mathrm{C}_{1}=\left(\mathrm{L}_{1}, \mathrm{~A}_{1}, \mathrm{~B}_{1}\right)$ and $\mathrm{C}_{2}=\left(\mathrm{L}_{2}, \mathrm{~A}_{2}, \mathrm{~B}_{2}\right)$, put:

$v\left(C_{1}, C_{2}\right)=\sqrt{\left(L_{1}-L_{2}\right)^{2}+\left(A_{1}-A_{2}\right)^{2}+\left(B_{1}-B_{2}\right)^{2}}$.

The consistency is then expressed as the sum of distances over all pairs - that is, the colour picked for the grapheme in the first versus second trial, first versus third trial, and second versus third trial. Denoting the colours as $C_{i}=\left(L_{i}, A_{i}, B_{i}\right)$ for $i$ $=1,2,3$, the sum is:

$\sum_{i \neq j \leq 3} v\left(C_{i}, C_{j}\right)=v\left(C_{1}, C_{2}\right)+v\left(C_{2}, C_{3}\right)+v\left(C_{3}, C_{1}\right)$.

Only the graphemes which were matched to a colour in all three trials within a round were included in the calculations. Overall within-round consistency score for each participant was then calculated as an arithmetic mean of the sums for each grapheme in the particular round. These scores enabled us to estimate the general consistency levels of the participants based on the cut-off value of 109.2 suggested by Rothen et al. (2013). Synaesthetes must score below this value to be classified as synaesthetic.

For the purposes of comparison between the rounds, we used CIEDE2000 distances, which is a standard metric for calculating distances in CIELAB space (Luo, Cui, \& Rigg, 2001; Sharma, Wu, \& Dalal, 2005). Only graphemes which were matched to a colour in each trial were included in this calculation. First, we computed mean CIEDE2000 distances between the colours of the three trials of each grapheme in each round (i.e. distance between Trial 1 and Trial 2, Trial 1 and Trial 3, and Trial 2 and Trial 3) using Formula 4: 
$d_{w}={ }^{1} / 3 \times \Delta E\left(C_{1}, C_{2}\right)+\Delta E\left(C_{2}, C_{3}\right)+\Delta E\left(C_{3}, C_{1}\right)$

where $C$ denotes a colour in CIELAB space and $d_{w}$ denotes the mean CIEDE2000 distance for a grapheme within a given round (i.e. the within-round distance).

Second, between-round distance score of a grapheme was calculated as a mean of all CIEDE2000 distances between each trial of each round, excluding the distances of colours picked in the same round. In other words, mean CIEDE2000 distance between each trial of a certain round and all other trials for the given grapheme in the two other rounds (i.e. the mean for these 27 distances) were computed using Formula 5:

$d_{b}={ }^{1} /{ }_{27} \times \sum_{i, j \leq 3} \sum_{k, l \leq 3, i<k} \Delta E\left(C_{i}^{j}, C_{k}^{l}\right)$,

where $C_{i}^{j}$ denotes the colour in CIELAB space picked in trial $j$ of round $i$.

\section{Results}

We report here only results of the 27 participants who completed all three rounds. Our data are freely accessible online (https://osf.io/u8nzd/).

First, the results for the short-term (within-round) consistency are presented. The main purpose is to assess that all participants may be classified as synaesthetes. Second, we present the results for long-term (between-round) consistency. This is the part which encompasses the main findings of the study. Third, we analyse the number of grapheme-colour associations and the differences in these numbers between the rounds.

Since the results for letter-colour and digit-colour associations are similar, we report only general results for grapheme-colour associations here (with an exception of the Overall Consistency for Participants section, where the general synaesthetic characteristics of the sample are introduced).

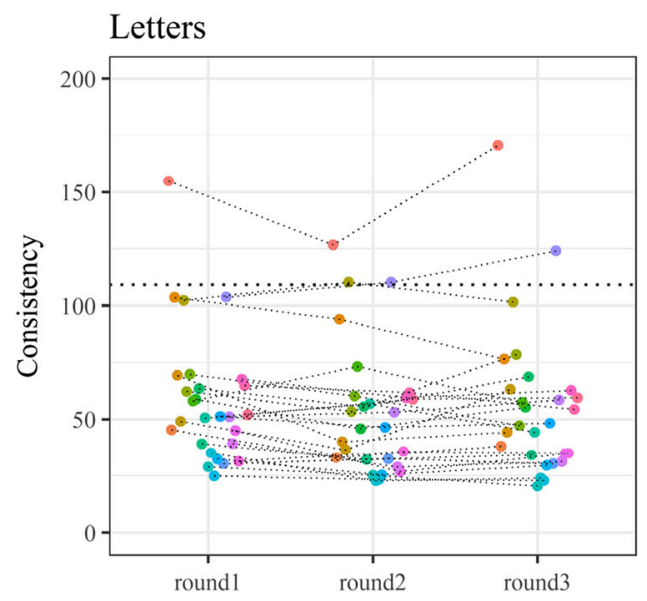

Detailed information about letter-colour and digit-colour associations may then be found in the Supplementary Materials.

\section{Short-term (within-round) consistency}

Overall consistency for participants The mean consistency scores for each speaker are presented in Fig. 1 (and in Tables S1 and S2 in the Supplementary Materials). In the first round, two participants scored above 109.2 on at least one test (letter-colour or digit-colour), exceeding the cut-off value for synaesthesia. These were Jessica for digit-colour test and Paul for letter-colour test. In the second round, three participants scored above 109.2 in the mean CIELAB score at least in one test (participants Paul, Shayla, and Emrie, all in the lettercolour test). In the third round, two participants scored above 109.2 (the participant Paul in both tests and the participant Emrie in the letter-colour test).

Because of the relatively high scores in all three rounds, we excluded Paul from further analyses because his status as a synaesthete is questionable. All the other 26 participants may be classified as synaesthetic based on most of the tests and based on CIELAB cut-off value 109.2, suggested by Rothen et al. (2013). In Table 1, the overall CIELAB consistency scores, together with their $95 \%$ confidence intervals for all the participants as a group, are presented. It may be seen that all the group scores are well below the cut-off value 109.2 (even the higher end of the interval).

\section{Long-term consistency}

Computer screens One of the possible problems of long-term consistency testing using the online battery is the possibility that different results in two tests may be caused by the difference between the computers (and their screens) used in the testing. Most of the participants (23 of 26) used the same computer according to their questionnaire responses, two participants (Patricia and Romy) used a different computer each

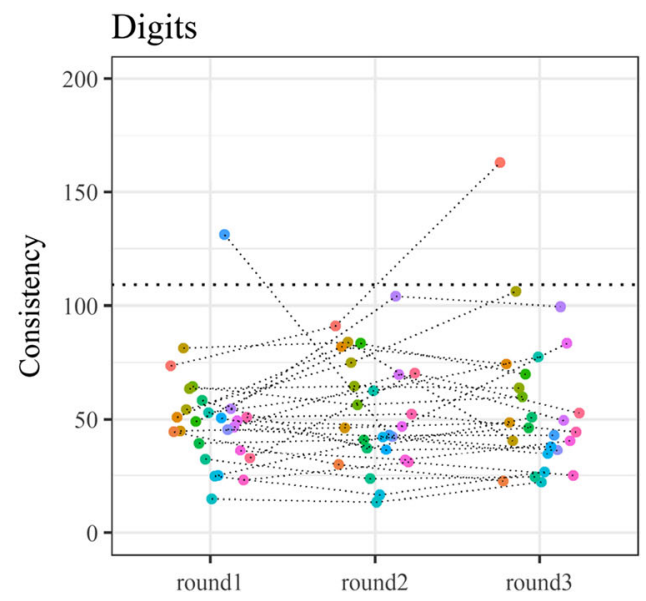

Fig. 1 Mean within-round consistency of grapheme-colour matchings for 27 participants who completed all three rounds. (Colour figure online) 
Table 1 Mean within-round CIELAB consistency scores of letters, numbers, and graphemes on the whole, together with the $95 \%$ confidence intervals for the 26 participants who completed all three rounds and were classified as synaesthetic

\begin{tabular}{llll}
\hline & Overall CIELAB score: Letters & Overall CIELAB score: Digits & Overall CIELAB score: Graphemes \\
\hline Round 1 & $55.07[51.88,58.27]$ & $48.58[42.94,54.23]$ & $53.73[50.94,56.52]$ \\
Round 2 & $50.5[47.55,53.45]$ & $51.43[44.94,57.92]$ & $50.69[48.00,53.38]$ \\
Round 3 & $52.16[49.06,55.26]$ & $51.14[45.07,57.22]$ & $51.96[49.20,54.72]$ \\
\hline
\end{tabular}

Note. The first value in each cell is the mean CIELAB consistency score; the values in square brackets denote $95 \%$ confidence intervals of the consistency score

time, and one participant (Amanda) used a different computer in the first round and in Rounds 2 and 3 because her former computer broke down between Rounds 1 and 2 and she had to buy a new one. These three participants are not excluded from the results, but we keep in mind that their possible inconsistency may be due to the inconsistencies in the colour display between the rounds.

Between-round consistency For the purposes of comparing within-round and between-round consistency, we used CIEDE2000 distances (the procedure was described above in the Analysis section).

In Fig. 2, the average between-round CIEDE2000 distance for each participant is plotted along with his or her three within-round scores. Precise numerical scores for each participant are shown in Table S3 in the Supplementary Materials.

To compare the between-round scores for each grapheme and participant with the three within-round scores, we used linear mixed effects modelling (Baayen, Davidson, \& Bates, 2008), which offers us a way for how to account for individual variation (on the level of both participants and graphemes).

The dependent variable was the CIEDE2000 distance score, and we tested the influence of two factors: (1) distance type (with four levels: Within-Round 1, Within-Round 2, WithinRound 3, and Between-Round), (2) participants' age (in years), and (3) ranked grapheme frequency in Czech (Bartoň, Cvrček, Čermák, Jelínek, \& Petkevič, 2009, pp. 118-119). As random effects, we used participant and grapheme.

Our main prediction was that the between-round distance should be significantly higher than all three other withinround distances. Because it has been shown that older people tend to have lower synaesthetic consistency levels (Meier et al., 2014; Simner et al., 2017), we included age as a factor into the model with a prediction that, the higher the participant's age, the higher the consistency score. The relevance of grapheme frequency for grapheme-colour synaesthesia has been analysed by Simner et al. (2005), who found that the grapheme frequency correlated with the frequency of associated colours and with the ease of generation of these colours. It may be possible that grapheme frequency is associated with colour consistency. Our prediction thus was that the lower the grapheme frequency, the lower the colour consistency.
Neither age nor ranked grapheme frequency yielded a significant effect in any model we created. The best model included distance type as the only fixed effect, and random intercepts for graphemes and participants, with random slopes for distance type. The model is described in Table 2 .

As can be seen from the model, the between-round score seems to be significantly higher than any of the within round scores (the $t$ value is well above 2 in all three cases).

Highly inconsistent cases The between-round CIEDE2000 scores have a skewed distribution, with the lowest score of 0.005 , highest score of 64.042 , and median of 12.317. We identified 55 cases of graphemes which could be characterized as outliers because their score is higher than 1.5 interquartile range from the median (i.e. higher than 36.85). Altogether, they were produced by 15 participants. Among the 55 cases, interesting colour changes between the rounds could be identified. For example, Jared matched the digit 7 twice to the "darksalmon"1 and once to the "lightsalmon2" colours in the first round, but to "aquamarine", "darkseagreen3", and "darkseagreen" in the second round. For the letter $E$, Susan chose colours "peachpuff" and "burlywood1" (twice) in the first round, "white" (twice), and "gray99" in the second round, and in the third round she picked "lightskyblue2" (twice) and "lightsteelblue2" for this letter. Sadie picked twice "slateblue2" and once "purple4" for the letter $\check{R}$ in the first round, "goldenrod1" (twice) and "goldenrod" in the second round, and "royalblue4" (twice) and "chocolate4" in the third round. Patricia matched the letter $F$ with "goldenrod2", "darkgoldenrod1", and "goldenrod1" in the first round, "chartreuse 3 " (three times) in the second round, and "olivedrab3" (twice), and "darkorange2" in the third round. Megan matched the digit 1 to "gray97", "gray94", and "gray95" in the first round; to "gray96", "gray94", and "gray97" in the second round; but to "firebrick3" (twice) and "red2" in the third round. Precise results for all the extreme cases may be found online (https://osf.io/u8nzd/).

\footnotetext{
${ }^{1}$ We are using colour names generated with "color.id" function in the package "plotrix" in R (Lemon, 2006).
} 


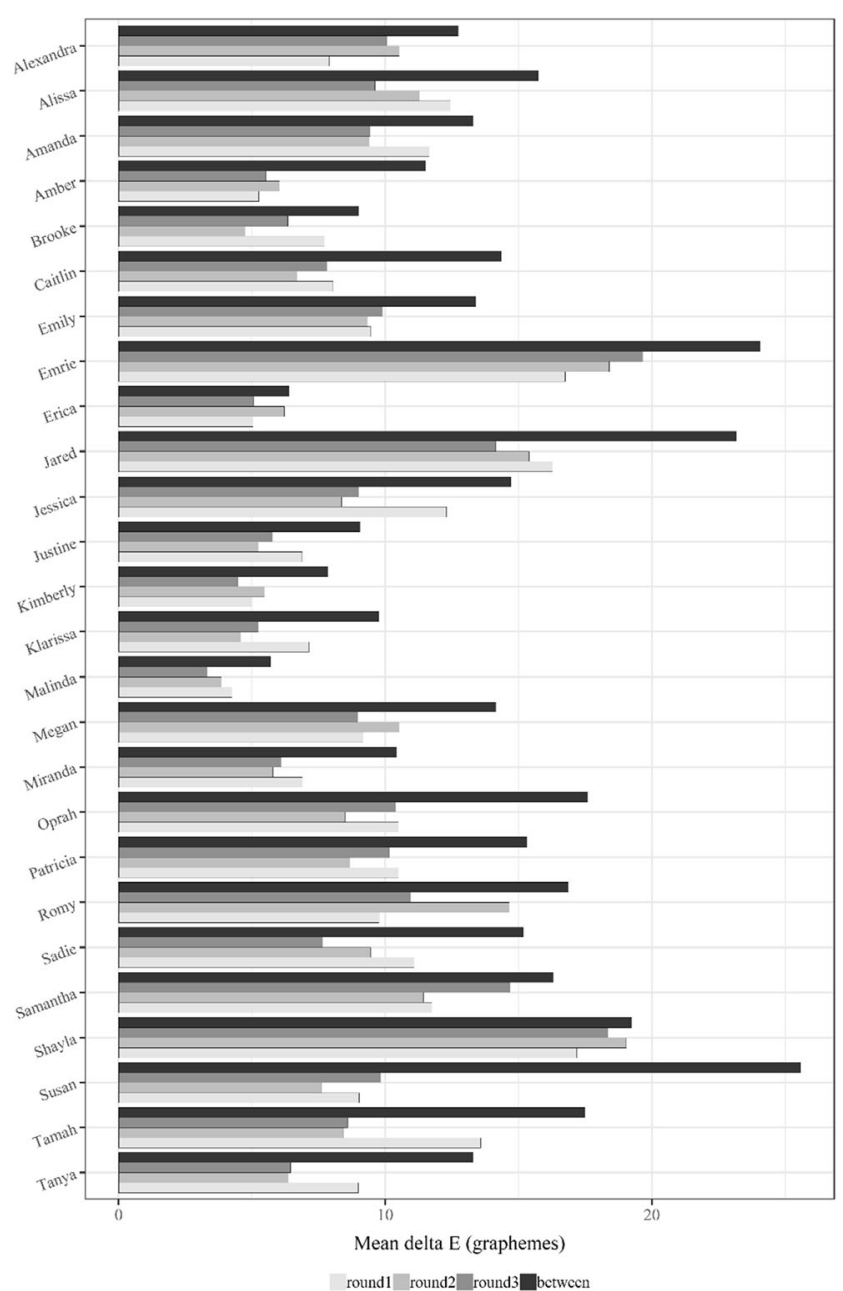

Fig. 2 Mean between-round CIEDE2000 distances and mean withinround CIEDE2000 distances for each participant who completed all three rounds (participant Paul was excluded due to his potentially nonsynaesthetic scores within all rounds)

Table 2 Linear-mixed model for CIEDE2000 distances, with distance type as a fixed effect (referential level: between round score) and two random effects: grapheme and participant (with distance type as random slope)

\begin{tabular}{lllll}
\hline Fixed effects & & Estimate & $S E$ & $t$ value \\
Intercept & & 14.334 & 1.016 & 14.111 \\
Distance type & Within-Round 1 & -4.541 & 0.61 & -7.44 \\
& Within-Round 2 & -5.255 & 0.693 & -7.588 \\
& Within-Round 3 & -5.186 & 0.618 & -8.396 \\
Random effects & & SD & & \\
Grapheme & & 1.415 & & \\
Participant & & 4.924 & & \\
& Within-Round 1 & 2.593 & & \\
& Within-Round 2 & 3.088 & & \\
& Within-Round 3 & 2.643 & & \\
Residual & & 8.193 & & \\
\hline
\end{tabular}

\section{Number of grapheme-colour associations}

The number of graphemes associated with a colour The number of grapheme-colour associations varied from 33 to 48 (out of 48 ) for all the participants and rounds. The mean was 46.29 and the median 48 . Thus, most of the participants matched colours to all graphemes (letters and digits) included in the battery. The precise number of graphemes associated with colours for each participant (separately for letters and digits) are presented in the Table S4 in the Supplementary Materials.

Surprisingly, not all participants matched the same number of graphemes to colours in each round. There were eight participants (Jessica, Oprah, Patricia, Romy, Samantha, Shayla, Susan, Tamah) whose number of grapheme-colour pairings differed between the rounds.

The number of trials with no association One possible reason for the inconsistency in the number of grapheme-colour pairings might be that the participants mistakenly used the "no colour" button instead of the "submit colour" button in one of the trials (unfortunately, the "no colour" button appeared just below the "submit colour" button on the screen). We can thus look at whether the graphemes which are not associated with a colour are not associated consistently in a given round or whether they are, since it is unlikely that an error of this type would occur more than once for each grapheme.

There were 45 cases of graphemes which differed between the rounds in whether or not they were matched with a colour by a particular participant. In 14 cases, we found that the colour was not submitted in one trial out of three within one round, and the colour was submitted three times in the other two rounds. There were three additional graphemes which were not submitted with a colour only once within a particular round, but they were also not submitted at least two times in a different round. In the remaining 27 cases, the colour was not submitted in two or three trials within a particular round. Four additional graphemes were not submitted only once in a certain round, but were submitted twice or three times in the next round.

Only Shayla's results could be generally interpreted as errors since five graphemes out of seven were not matched with a colour in only one trial. With other participants, the number of graphemes they did not match to a colour only once was smaller than, or equal to, the number of letters they did not match in two or three trials. In other words, it seems that the between-round differences in graphemes which were and were not matched are probably not due to an error during the testing phase.

It is worth pointing out that three of the above-mentioned participants (viz. Patricia, Romy, and Tamah) matched all graphemes to colours in the second and the third round but not in the first. We will come back to that in the Discussion. 


\section{Discussion}

The aim of this study was to systematically examine the stability of grapheme-colour matchings in the adult population. We presented a method of measurement of the long-term consistency using standard computerized tests. We showed that the colours associated with graphemes in grapheme-colour synaesthesia can change substantially in adults (for example, from salmon to aquamarine, from gold to chartreuse). Based on a linear mixed model, we argue that short-term and long-term consistency should be distinguished, since these two scores differ significantly (see Long-Term Consistency section). It should be emphasized that we documented these changes within a relatively short time frame of 1 year and a half. Thus, it remains an open question to what extent the colours change over longer time periods, such as decades, which is the time frame targeted by other studies relating synaesthetic consistency and age (Meier et al., 2014; Simner et al., 2017).

In our data, we found no age-related effects on consistency. However, the sample was not designed for testing age effects. Only four participants were older than 35 years when they took part in the first round of the research. Therefore, we are far from inferring any strong conclusions about the role of age in grapheme-colour consistency.

We documented several examples of graphemes that were not matched to a colour by a synaesthete in one round, but were matched (consistently) in a different round. A possible interpretation could be that this is due to technical reasons. It might be that the participants mistakenly used the "no colour" button instead of the "submit colour" button in one of the trials, since the "no colour" button appeared just below the "submit colour" button on the screen. We tested this possibility in the section Number of Grapheme-Colour Associations and found out that in more than half of the given cases, the colour was not submitted in two or three trials within a round. Therefore, it seems very likely that these cases are not due to an error during the testing phase. We may thus only speculate what is the reason for these cases. One possible interpretation is that the vividness of certain grapheme-colour associations is rather low for the given synaesthetes, and thus they may have problems ascribing the colour in a particular time point. This possibility is supported by self-reports: Some of our participants claimed that certain graphemes had a more vivid colour for them than others, and that their response was "easier" for the more vivid graphemes. Interestingly, one of the participants who completed only the first round told us that it had been almost impossible for her to match the graphemes to colours adequately, since the palette in the battery she had picked from strongly interfered with her grapheme-colour experiences. Low vividness could also be why certain people who claim to have synaesthetic experiences fail in the consistency tests (cf. Simner, 2012a).

We were confronted with three serious problems while conducting this study. One was sample attrition, which is rather typical of panel studies (Lynn, 2009, p. 10). Although only 27 participants from the initial sample of 42 completed all three rounds, we believe that the sample size of 27 participants is appropriate for this kind of research for at least two reasons: (1) The overall prevalence of this kind of synaesthesia is very low (i.e. between $1 \%$ and $2 \%$ of the general population; cf. Carmichael et al., 2015; Simner et al., 2006), and therefore it is extremely difficult to get a substantially larger sample. (2) The purpose of the analysis is not to make any far-reaching generalizations for the whole synaesthetic population but rather to show whether grapheme-colour matchings can change in time and whether it should be distinguished between long-term and short-term consistency. For these purposes, an even smaller sample should be sufficient.

A second problem was associated with the fact that three participants (Amanda, Patricia, and Romy) did not use the same computer in all three rounds. This may bias their results towards higher between-round inconsistency since different computers may display colours differently. Importantly, the between-round consistency scores for the three of them were not significantly higher than scores of other participants, so we suggest that the use of different screens did not cause a distortion in our results.

A third problem lies in the fact that we did not control the lighting conditions of the testing. However, the highly inconsistent cases we report can hardly be explained by differences in lighting conditions amongst the rounds.

Our study may also be affected by the choice of The Synaesthesia Battery (Eagleman et al., 2007) as a testing tool. Although this battery is currently the most widely used tool for synaesthesia testing, there are several issues which we saw as unnecessary complications. First, the battery offers the participants their results, with a commentary telling them whether they are synaesthetic or not based on the rigorous cut-off value of 1 in the RGB score. This seems to us to be theoretically unfounded and misleading (cf. Eagleman, 2012; Simner, 2012a). We are convinced that this partly caused the sample attrition since eight of the 14 participants who did not continue after Round 1 scored above 1 in the battery and were told by the battery that they were not considered as synaesthetic. It is likely that they lost their interest and confidence in our research afterwards. The second problem with the battery is the already mentioned closeness of the "submit colour" and "no colour" buttons (see Number of Grapheme-Colour Associations section). It seems likely that at least some cases of "no colour" responses were mistakes. This cannot be fully dealt with, but we developed a way of estimating whether the "no colour" responses were in fact errors, since it is unlikely that a participant will make the same mistake two or three times for a given grapheme.

However, we fully subscribe to Simner's (2012a, 2012b) view that the definitions of synaesthesia should not rely on consistency and that synaesthesia may not be limited to consistent cases. In this article, we support this argument with evidence that grapheme-colour associations can change over time, even in 
synesthetes which would be classified as consistent at the 109.2 CIELAB cut-off value suggested by Rothen et al. (2013). We believe that future research on synaesthesia should consider not only short-term consistent synaesthetes but also those who fail the consistency tests but claim to have synaesthetic experiences.

\section{Conclusions}

We believe that our study presents convincing evidence that grapheme-colour matchings can indeed change during the lifetime, and that there are certain issues which synaesthesia research should be wary of in the future, such as the use of confidence intervals, differences in the vividness for colours ascribed to different graphemes by a particular participant, and the general difference between short-term and long-term consistency.

Funding This study was supported by the Charles University project PROGRES 4, Language in the Shiftings of Time, Space, and Culture.

\section{References}

Asher, J. E., Aitken, M. R. F., Farooqi, N., Kurmani, S., \& Baron-Cohen, S. (2006). Diagnosing and phenotyping visual synaesthesia: A preliminary evaluation of the revised Test of Genuineness (TOG-R). Cortex, 42(2), 137-146. https://doi.org/10.1016/S0010-9452(08) 70337-X

Baayen, R. H., Davidson, D. J., \& Bates, D. M. (2008). Mixed-effects modeling with crossed random effects for subjects and items. Journal of Memory and Language, 59(4), 390-412.

Baron-Cohen, S. (1996). Is there a normal phase of synaesthesia in development. Psyche, 2(27), 223-228.

Baron-Cohen, S., Harrison, J., Goldstein, L. H., \& Wyke, M. (1993). Coloured speech perception: Is synaesthesia what happens when modularity breaks down?. Perception, 22(4), 419-426.

Bartoň, T., Cvrček, V., Čermák, F., Jelínek, T., \& Petkevič, V. (2009). Statistiky češtiny [Czech statistics]. Prague: Nakladatelství Lidové noviny.

Carmichael, D. A., Down, M. P., Shillcock, R. C., Eagleman, D. M., \& Simner, J. (2015). Validating a standardised test battery for synaesthesia: Does the Synaesthesia Battery reliably detect synaesthesia? Consciousness and Cognition, 33, 375-385. https://doi.org/10. 1016/j.concog.2015.02.001

Dixon, M. J., Smilek, D., \& Merikle, P. M. (2004). Not all synaesthetes are created equal: Projector versus associator synaesthetes. Cognitive, Affective, \& Behavioral Neuroscience, 4(3), 335-343. https://doi.org/10.3758/CABN.4.3.335

Eagleman, D. M. (2012). Synaesthesia in its protean guises. British Journal of Psychology, 103(1), 16-19. https://doi.org/10.1111/j. 2044-8295.2011.02020.x

Eagleman, D. M., Kagan, A. D., Nelson, S. S., Sagaram, D., \& Sarma, A. K. (2007). A standardized test battery for the study of synaesthesia. Journal of Neuroscience Methods, 159(1), 139-145. https://doi.org/ 10.1016/j.jneumeth.2006.07.012

Gama, J., \& Davis, G. (2018). colorscience: Color Science Methods and Data (R Package Version 1.0.5) [Computer software]. Retrieved from https://CRAN.R-project.org/package=colorscience/
Kay, C., Carmichael, D., Ruffell, H., \& Simner, J. (2015). Colour fluctuations in grapheme-colour synaesthesia: The effect of clinical and non-clinical mood changes. British Journal of Psychology, 106, 487-504. https://doi.org/10.1111/bjop.12102

Lemon, J. (2006). Plotrix: A package in the red light district of R. $R$ News, 6(4), 8-12.

Luo, M. R., Cui, G., \& Rigg, B. (2001). The development of the CIE 2000 colour-difference formula: CIEDE2000. Color Research \& Application, 26(5), 340-350. https://doi.org/10.1002/col.1049

Lynn, P. (2009). Methods for longitudinal surveys. In P. Lynn (Ed.), Methodology of longitudinal surveys (pp. 1-19). Chichester: Wiley.

Meier, B., Rothen, N., \& Walter, S. (2014). Developmental aspects of synaesthesia across the adult lifespan. Frontiers in Human Neuroscience, 8(129). https://doi.org/10.3389/fnhum.2014.00129

Rich, A. N., Bradshaw, J. L., \& Mattingley, J. B. (2005). A systematic, large-scale study of synaesthesia: Implications for the role of early experience in lexical-colour associations. Cognition, 98(1), 53-84. https://doi.org/10.1016/j.cognition.2004.11.003

Rothen, N., Seth, A. K., Witzel, C., \& Ward, J. (2013). Diagnosing synaesthesia with online colour pickers: Maximising sensitivity and specificity. Journal of Neuroscience Methods, 215(1), 156-160. https://doi.org/10.1016/j.jneumeth.2013.02.009

Sharma, G., Wu, W., \& Dalal, E. N. (2005). The CIEDE2000 colordifference formula: Implementation notes, supplementary test data, and mathematical observations. Color Research \& Application, 30(1), 21-30. https://doi.org/10.1002/col.20070

Simner, J. (2007). Beyond perception: synaesthesia as a psycholinguistic phenomenon. Trends in Cognitive Sciences, 11(1), 23-29. https:// doi.org/10.1016/j.tics.2006.10.010

Simner, J., Ipser, A., Smees, R., \& Alvarez, J. (2017). Does synaesthesia age? Changes in the quality and consistency of synaesthetic associations. Neuropsychologia, 106, 407-416. https://doi.org/10.1016/j. neuropsychologia.2017.09.013

Simner, J. (2012a). Defining synaesthesia. British Journal of Psychology, 103(1), 1-15. https://doi.org/10.1348/000712610X528305

Simner, J. (2012b). Defining synaesthesia: A response to two excellent commentaries. British Journal of Psychology, 103(1), 24-27. https://doi.org/10.1111/j.2044-8295.2011.02059.x

Simner, J., Harrold, J., Creed, H., Monro, L., \& Foulkes, L. (2009). Early detection of markers for synaesthesia in childhood populations. Brain, 132(1), 57-64. https://doi.org/10.1093/brain/awn292

Simner, J., Mulvenna, C., Sagiv, N., Tsakanikos, E., Witherby, S. A., Fraser, C., ... Ward, J. (2006). Synaesthesia: The prevalence of atypical cross-modal experiences. Perception, 35(8), 1024-1033. https://doi.org/10.1068/p5469

Simner, J., Ward, J., Lanz, M., Jansari, A., Noonan, K., Glover, L., \& Oakley, D. A. (2005). Non-random associations of graphemes to colours in synaesthetic and non-synaesthetic populations. Cognitive Neuropsychology, 22(8), 1069-1085. https://doi.org/10. 1080/02643290500200122

Ward, J., Li, R., Salih, S., \& Sagiv, N. (2007). Varieties of graphemecolour synaesthesia: A new theory of phenomenological and behavioural differences. Consciousness and Cognition, 16(4), 913-931. https://doi.org/10.1016/j.concog.2006.09.012

Ward, J., \& Simner, J. (2005). Is synaesthesia an X-linked dominant trait with lethality in males? Perception, 34(5), 611-623. https://doi.org/ $10.1068 / \mathrm{p} 5250$

Watson, M. R., Chromý, J., Crawford, L., Eagleman, D. M., Enns, J. T., \& Akins, K. A. (2017). The prevalence of synaesthesia depends on early language learning. Consciousness and Cognition, 48, 212231. https://doi.org/10.1016/j.concog.2016.12.004

Publisher's note Springer Nature remains neutral with regard to jurisdictional claims in published maps and institutional affiliations. 